\title{
Development of a Mobile App for Individuals with Co-Occurring Substance Use and Mood Disorders: Integrated Support Now
}

Dawn Sugarman ${ }^{1,2^{*}}$, PhD; Sunetra Bane ${ }^{3 *}$; Simone Orlowski ${ }^{2,3,4}, \mathrm{PhD}$; Tasmia Noor ${ }^{3}$; Joe Gracz ${ }^{3}$; Hilary Connery ${ }^{1,2}$, MD, PhD; Cheryl Cronin ${ }^{1}$; Kenneth Gilman ${ }^{1}$; Rocco Iannucci ${ }^{1,2}, \mathrm{MD}$; Monika Kolodziej ${ }^{1,2}$, PhD; Anna Munro ${ }^{1,2}$, LICSW; Kamal Jethwani ${ }^{2,3,4}$, MD, MPH; Roger Weiss ${ }^{1,2}$, MD

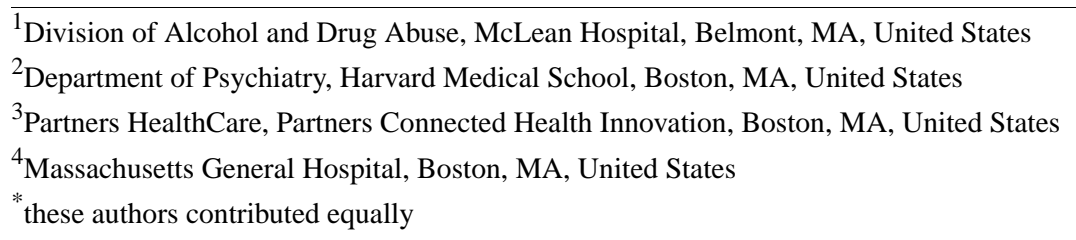

Corresponding Author:

Dawn Sugarman, PhD

Division of Alcohol and Drug Abuse

McLean Hospital

115 Mill Street

Belmont, MA,

United States

Phone: 8553650

Email: dsugarman@mclean.harvard.edu

\section{Abstract}

Background: Mood disorders and substance use disorders (SUDs) co-occur at a high rate. Individuals with co-occurring mood and substance use disorders are less likely to complete treatment. Of those who receive treatment, many do not receive adequate care that addresses both disorders. Integrated Group Therapy (IGT) is an evidence-based psychosocial treatment that treats both mood disorders and SUDs, stressing the similarities and relationship between the two disorders. Although IGT is an effective treatment for individuals with mood and substance use disorders, it is not widely available, and for those who do receive IGT, there is no in-the-moment support.

Objective: A mobile version of IGT would increase access and provide in-the-moment support for individuals when they need it. The aim of this study is to use exploratory, qualitative user-centered design methodology to interview and observe end users and clinicians who treat individuals with mood disorders and SUDs to inform the design of the mobile app.

Methods: Qualitative interviews were conducted with 5 patient participants who were currently receiving treatment for a co-occurring mood and substance use disorder, and 5 clinicians with experience treating patients with mood disorders and/or substance use disorders. All participants completed a short survey to assess demographic information and technology use. Additionally, observations were conducted at 3 IGT inpatient and outpatient groups to triangulate findings across methods. Interviews were audio-recorded and transcribed. Transcripts and field notes were analyzed using thematic analysis using NVivo for Mac (version 11).

Results: Patient participants were predominately male $(3 / 5,60 \%)$, age $45-64(4 / 5,80 \%)$, unemployed or disabled $(3 / 5$, $60 \%)$, and white $(5 / 5,100 \%)$. The majority of clinicians were female $(4 / 5,80 \%)$, age $26-44(5 / 5,100 \%)$, and white $(4 / 5,80 \%)$. Most patients $(4 / 5,80 \%)$ and clinicians $(5 / 5,100 \%)$ reported feeling comfortable using technology as a treatment tool, and $40 \%(2 / 5)$ of patients indicated that they had experience doing so. Key treatment themes that emerged from the qualitative data included the importance of IGT in helping patients to develop a common language to describe their co-occurring conditions and experiences, visualizing the recovery journey, the importance of independence and freedom to patients throughout treatment, along with varied acceptance and self-perception of one's recovery journey. With respect to developing a mobile tool, reported patient needs included: in-the-moment support, peer-to-peer support, after-care planning, maintaining structure post-discharge from treatment and opportunities to practice skills. Clinicians corroborated the need for patient peer-to-peer support, help with after-care planning and the opportunity to practice skills. 
Conclusions: Patients and clinicians were open to the idea of using technology as part of treatment. Several themes emerged to inform the direction of a minimal viable product (MVP) of the app. Next steps include narrowing down to key themes to focus on for the MVP, defining features as relevant to those themes, designing a clickable prototype of the app and conducting iterative feedback sessions with end users.

(iproc 2018;4(2):e11892) doi: $\underline{10.2196 / 11892}$

\section{KEYWORDS}

addiction; mobile health intervention; mood disorders

Edited by T Hale; this is a non-peer-reviewed article. Submitted 09.08.18; accepted 29.08.18; published 17.09.18.

Please cite as:

Sugarman D, Bane S, Orlowski S, Noor T, Gracz J, Connery H, Cronin C, Gilman K, Iannucci R, Kolodziej M, Munro A, Jethwani $K$, Weiss $R$

Development of a Mobile App for Individuals with Co-Occurring Substance Use and Mood Disorders: Integrated Support Now iproc 2018;4(2):e11892

URL: http://www.iproc.org/2018/2/e11892/

doi: $10.2196 / 11892$

PMID:

(CDawn Sugarman, Sunetra Bane, Simone Orlowski, Tasmia Noor, Joe Gracz, Hilary Connery, Cheryl Cronin, Kenneth Gilman, Rocco Iannucci, Monika Kolodziej, Anna Munro, Kamal Jethwani, Roger Weiss. Originally published in Iproceedings (http://www.iproc.org), 17.09.2018. This is an open-access article distributed under the terms of the Creative Commons Attribution License (https://creativecommons.org/licenses/by/4.0/), which permits unrestricted use, distribution, and reproduction in any medium, provided the original work, first published in Iproceedings, is properly cited. The complete bibliographic information, a link to the original publication on http://www.iproc.org/, as well as this copyright and license information must be included. 